\title{
Collocation of geostationary satellites using convex optimization
}

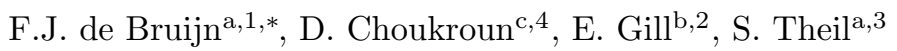 \\ ${ }^{a}$ German Aerospace Center (DLR), Institute of Space Systems, Robert-Hooke-Strasse 7, 28359, Bremen, Germany \\ ${ }^{b}$ Delft University of Technology, Faculty of Aerospace Engineering, Kluyverweg 1, 2629 HS, Delft, Netherlands \\ ${ }^{c}$ Ben-Gurion University of the Negev, Beer-Sheva, 84105, Israel
}

\begin{abstract}
A method is developed to calculate station-keeping maneuvers for a fleet of satellites collocated in a geostationary slot. The method is enabled by linear time-varying formulation of the dynamics in terms of non-singular orbital elements. A leader-follower control hierarchy is used, so that the motion of the follower satellites is controlled relative to the leader. Key objectives of the station-keeping problem are a minimization of propellant consumption, using a limited number of maneuvers, while guaranteeing safe separation between the satellites. The method is applied in a realistic simulation scenario, including orbit determination, actuation and modeling errors. The method is demonstrated to work for an inhomogeneous fleet of four satellites with a maneuver cycle of one week. It is then demonstrated that by reducing the maneuver cycle duration to one day, it is possible to collocate 16 satellites in a single slot, without penalties on propellant consumption.
\end{abstract}

Keywords: Collocation, geostationary satellites, convex optimization, leader/follower, station-keeping

\section{Introduction}

Syncom II was the first satellite to arrive in a geosynchronous orbit. Since that time many satellites followed, and in particular the geostationary orbit has become increasingly populated. Driven by the need to avoid radiofrequency interference between different satellites, the geostationary orbit was divided into slots, which are allocated by the International Telecommunication Union. The limited availability and difficulty of obtaining these slots, especially at key locations above highly populated areas, together with the ever increasing need for geostationary satellite services lead several organizations to collocate multiple satellites within a geostationary slot, see e.g. [1].

Collocation strategies used to control more than two satellites in one slot generally rely on a coordinated approach to specifying desired states. The satellites' desired mean eccentricity and inclination vectors are defined in a configuration that is passively safe. Each satellite in the fleet is then controlled individually to stay close to this desired state [2]. The idea is to maintain relative eccentricity and inclination vectors (anti-)parallel, to ensure that radial separation is maximum when normal separation van-

\footnotetext{
*Corresponding author

Email addresses: Frederik.Bruijn@DLR.de (F.J. de Bruijn), danielch@bgu.ac.il (D. Choukroun), E.K.A.Gill@tudelft.nl (E Gill)

${ }^{1}$ Researcher, Department of GNC systems

${ }^{2}$ Professor and Chairholder, Department of Space Systems

${ }^{3}$ Department Head GNC systems

${ }^{4}$ Guest Assistant-Professor, Department of Space Systems, Delft University of Technology
}

ishes and vice-versa. This principle is called eccentricity/inclination vector separation. Tangential separation is usually ignored, since the satellite's along-track position is very sensitive to orbit determination and maneuver errors, and hence, the uncertainty associated to the along-track position is large for long prediction horizons (i.e. [3] reports a $4 \mathrm{~km}(3 \sigma)$ uncertainty for 7 day prediction horizon due to orbit determination errors only).

Since the satellites are controlled individually, and osculating eccentricity and inclination vectors show relatively large variations even within one orbit, the control windows in which the eccentricity and inclination vectors are to be controlled are normally large. The geostationary slot boundaries limit the configuration space within which the eccentricity and inclination vectors can vary. Strategies such as sun-pointing perigee further limit the available configuration space. Thus the size of the geostationary slot, the control windows on the eccentricity and inclination vectors, and the station-keeping strategy together limit the number of satellites that can be maintained within a geostationary slot.

In [4] it is demonstrated that differential perturbations between satellites are small within a geostationary slot. This suggests that, in the absence of maneuvers, variations in the osculating relative states are small as well. If we can formulate the guidance and control problem directly in terms of osculating relative states, we are able to maintain these relative states within small tolerance windows. This allows to increase the number of satellites that are collocated within a single slot.

This paper develops a method to accurately control 
the relative orbital elements of collocated geostationary satellites. We show that by controlling relative orbital elements, most notably inclination and eccentricity vectors, we indirectly control (minimum) separation distances between satellites. To this end the method for stationkeeping of geostationary satellites introduced in [4] is extended to take into account constraints on relative states. A leader-follower control hierarchy is used to control the relative states. The leader satellite can be controlled using any desired method (we use the method from [4]), and it is assumed that the leader's state trajectory is available for determining the followers' station-keeping maneuvers. These maneuvers are determined by formulating and solving a convex optimization problem. The method is further improved by explicitly accounting for the thruster configuration and using directly the thrusts of each individual thruster as independent variables in the optimization problem. The optimization problem is scaled to improve the numeric solution.

A simulation campaign was performed and the method is demonstrated to work under realistic (even conservative) orbit determination errors, actuator errors and modeling errors, and for satellites with different characteristics. It is also shown that by decreasing the maneuver cycle duration, it is possible to collocate a greater number of satellites in a slot.

A demonstration of the method is given for four satellites, for a maneuver cycle duration of 7 days, and for a fleet of 16 satellites for a maneuver cycle duration of 1 day. Results are analyzed in terms of propellant consumption, number of maneuvers, accuracy of control of relative orbital elements, and minimum separation distances.

Corollary results include that propellant savings are achieved by explicitly accounting for thruster configuration in the station-keeping strategy. It is shown that the classical sun-pointing perigee strategy is not required for typical electric propulsion thruster configurations in order to save propellant. And lastly, the thruster configuration itself has significant impact on achieved control accuracy.

\section{Development of Dynamics}

\subsection{Review of Dynamics}

The theory described in this section builds on foundations from [4]. A short review of the key concepts is given here. A linear time-varying formulation of the dynamics of a satellite in a geostationary orbit is obtained through two simplifying assumptions:

Assumption 1 The input matrix at the ideal GEOslot center provides a good approximation of the input matrix at an arbitrary position inside the GEO-slot.

A similar assumption is applied to the perturbing accelerations:

Assumption 2 The differential accelerations (excluding controlled accelerations) between a satellite at an arbitrary position inside a GEO-slot and a virtual satellite located at the GEO-slot center are at all times small.

These assumptions were investigated in [4] and bounds on the maximum error resulting from these assumptions were given. The assumptions allowed to write the dynamics of a geostationary satellite as a linear time-varying system:

$$
\dot{\mathbf{x}}(t) \approx \mathbf{A x}(t)+\mathbf{B}(\alpha) \mathbf{u}_{c}(t)+\mathbf{B}(\alpha) \mathbf{u}_{d}\left(\mathbf{x}_{\mathrm{geo}}\right) .
$$

The state is defined in the orbital element set introduced in [5] and is as follows:

$$
\mathbf{x}(t)=\left(\begin{array}{c}
\Delta n \\
e_{y} \\
e_{x} \\
i_{y} \\
i_{x} \\
\Delta L
\end{array}\right)=\left(\begin{array}{c}
n-n_{E} \\
e \sin (\tilde{\omega}) \\
e \cos (\tilde{\omega}) \\
\sin \left(\frac{i}{2}\right) \sin (\Omega) \\
\sin \left(\frac{i}{2}\right) \cos (\Omega) \\
\varepsilon+\int_{t_{0}}^{t} n \mathrm{~d} t-\alpha
\end{array}\right)
$$

where $n=\sqrt{\mu / a^{3}}$ is the mean orbital motion, $n_{E}$ is the Earth's rotation rate, $\tilde{\omega}=\omega+\Omega, \varepsilon$ is the mean longitude at epoch $t_{0}, \alpha$ is the right ascension of the geostationary position and $a, e, i, \omega, \Omega$ are classical orbital elements. The A matrix contains all zeros, except the $(6,1)^{\text {th }}$-entry, which is equal to one. The input matrix $\mathbf{B}$ is evaluated at the geostationary slot center:

$$
\mathbf{B}(\alpha) \approx\left(\begin{array}{ccc}
0 & \frac{-3}{a_{\text {geo }}} & 0 \\
-\frac{1}{V_{\text {geo }}} \cos \alpha & \frac{2}{V_{\text {geo }}} \sin \alpha & 0 \\
\frac{1}{V_{\text {geo }}} \sin \alpha & \frac{2}{V_{\text {geo }}} \cos \alpha & 0 \\
0 & 0 & \frac{1}{2 V_{\text {geo }}} \sin \alpha \\
0 & 0 & \frac{1}{2 V_{\text {geo }}} \cos \alpha \\
-\frac{2}{V_{\text {geo }}} & 0 & 0
\end{array}\right),
$$

where $V_{\text {geo }}$ and $a_{\text {geo }}$ are respectively velocity and semimajor axis of an ideal geostationary satellite and the right ascension of the slot center is determined from:

$$
\alpha(t)=\alpha\left(t_{0}\right)+n_{E}\left(t-t_{0}\right),
$$

The perturbing accelerations $\mathbf{u}_{d}\left(\mathbf{x}_{\text {geo }}\right)$ are determined at the slot center $\left(\mathbf{x}_{\text {geo }}\right)$ and both perturbing and controlled accelerations $\mathbf{u}_{c}(t)$ are defined in a radial, tangential, normal reference frame.

In summary, the dynamics of a satellite relative to the geostationary slot center can be described by a linear timevarying system, driven by a perturbing acceleration $\mathbf{u}_{d}(t)$. References for obtaining an expression for the perturbing accelerations are ample, see e.g. [6] or [7]. Both the perturbing acceleration and the time-varying input matrix $B(t)$ are, for a given geostationary slot, non-linear but known function of time (under Assumptions 1 and 2). 
Equation 1 is discretized using a zero-order hold on the control input $\mathbf{u}_{c}(t)$, while applying the classic RungeKutta 4 scheme to obtain the discrete update:

$$
\mathbf{x}_{k+1}=\tilde{\mathbf{A}} \mathbf{x}_{k}+\tilde{\mathbf{B}}_{k} \mathbf{u}_{k}+\tilde{\mathbf{d}}_{k}
$$

where $k$ refers to the $k^{\text {th }}$ discrete step, $\tilde{\mathbf{A}}, \tilde{\mathbf{B}}_{k}$ and $\tilde{\mathbf{d}}_{k}$ are obtained by applying RK4 method to Eq. 1 for a certain timestep $h$. An explicit definition of the matrices is given in $[4]$.

If we now repeatedly apply Eq. 5 from $k=0$ to $k=$ $N-1$, we can obtain a linear equation,

$$
X=\mathbf{F} \mathbf{x}_{0}+\mathbf{H} U+\mathbf{J} D,
$$

that relates the (concatenated) $N$ future states $X=$ $\left(\mathbf{x}_{1}^{T}, \mathbf{x}_{2}^{T}, \ldots, \mathbf{x}_{N}^{T}\right)^{T}$ to the current state $\mathbf{x}_{0}$ and the (concatenated) controls $U=\left(\mathbf{u}_{0}^{T}, \mathbf{u}_{1}^{T}, \ldots, \mathbf{u}_{N-1}^{T}\right)^{T}$.

Disturbances $D=\left(\tilde{\mathbf{d}}_{0}^{T}, \tilde{\mathbf{d}}_{1}^{T}, \ldots, \tilde{\mathbf{d}}_{N-1}^{T}\right)^{T}$ and matrices $\mathbf{F}$, $\mathbf{H}$ and $\mathbf{J}$ follow from a repeated application of Eq. 5 .

The development of this approach is extensively treated in [4] and explicit definitions of matrices are given there. The key feature of this formulation of the dynamics is that it gives us an affine expression relating the osculating future states to the osculating current state and future controls, without neglecting the perturbing accelerations, and it is this feature that enables us to solve problems of station-keeping and collocation using convex optimization techniques.

\subsection{Thruster configuration}

Most common geostationary satellite are three-axis stabilized platforms using chemical propulsion thrusters. These thrusters are generally aligned with North, East, South and West directions. This ties in well with the dynamics, since the controlled accelerations are defined in radial, tangential and normal direction of the orbital reference frame (n.b. tangential and normal direction correspond to respectively East/West and North/South directions).

Satellites employing an electric propulsion system usually have a different layout. North and South facing panels are home to solar panels, and the plumes originating from an electric thruster can cause significant degradation to the solar panel. Hence, the thrusters are generally tilted with respect to the North and South directions to avoid contamination. To illustrate this, we define a thruster configuration with four thrusters. Thrusters one and four point at an angle $\gamma \in\left[0,90^{\circ}\right]$ away from the North, while two and three point at an angle $\gamma \in\left[0,90^{\circ}\right]$ away from the South. Furthermore, thrusters one and two point at an angle $\beta \in\left[0,90^{\circ}\right]$ away from the radial direction towards the East and three and four at an angle $\beta \in\left[0,90^{\circ}\right]$ away from the radial direction towards the West. One configuration (A) used in this paper is obtained for $\gamma=45^{\circ}$ and $\beta=90^{\circ}$ (Fig. 1). The arrows point in the direction of

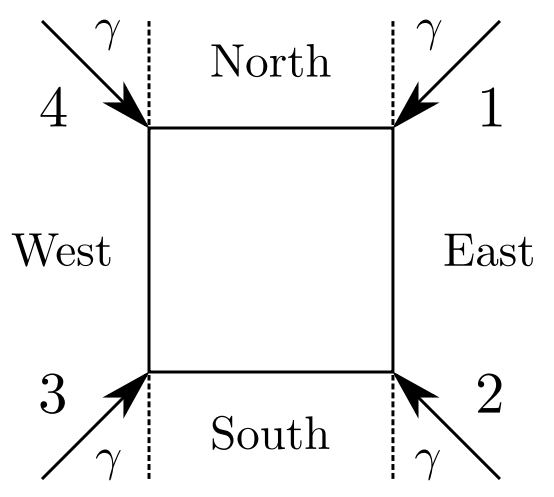

Figure 1: Exemplary electric propulsion thruster configuration

the acceleration that is exerted on the spacecraft by each thruster (which is opposite to the exhaust plume). With this choice for $\beta$ and $\gamma$ the thrust vector lies completely in the tangential, normal plane, which is a common choice on geostationary satellites. This configuration is similar as implemented on the Hispasat Advanced Generation 1 mission [8]. Another configuration (B) that is analysed in this paper is obtained by choosing $\gamma=45^{\circ}$ and $\beta=10^{\circ}$. Note that for this configuration the thrusters are both pointing away from the solar panels and from the Earth-facing panel. We also use a reference configuration (REF) with four thrusters pointing respectively North, East, South and West.

The thruster configuration can be taken into account in the dynamics formulation by employing a matrix that maps the individual thrust directions to accelerations in the radial, tangential, normal plane:

$$
\mathbf{u}=\frac{1}{m} \Gamma \mathbf{T}
$$

where $m$ is the satellite mass, $\mathbf{T}=\left(T_{1}, T_{2}, T_{3}, T_{4}\right)^{T}$ is the vector of individual thrusts and $\Gamma$ is the thruster configuration matrix, for our definition of $\beta$ and $\gamma$ we obtain:

$\Gamma=\left(\begin{array}{cccc}-\sin \gamma \cos \beta & -\sin \gamma \cos \beta & -\sin \gamma \cos \beta & -\sin \gamma \cos \beta \\ -\sin \gamma \sin \beta & -\sin \gamma \sin \beta & \sin \gamma \sin \beta & \sin \gamma \sin \beta \\ -\cos \gamma & \cos \gamma & \cos \gamma & -\cos \gamma\end{array}\right)$

This can be used to reformulate Eq. 6 to:

$$
X=\mathbf{F} \mathbf{x}_{0}+\frac{1}{m} \mathbf{H} \boldsymbol{\Gamma} T+\mathbf{J} D
$$

with

$$
\boldsymbol{\Gamma}=\left(\begin{array}{ccc}
\Gamma & & \\
& \Gamma & \\
& & \ddots
\end{array}\right) .
$$

and $T=\left(\mathbf{T}_{0}^{T}, \mathbf{T}_{1}^{T}, \ldots, \mathbf{T}_{N-1}^{T}\right)^{T}$. This formulation relates directly the thrusts by each thruster to the satellite state, described in non-singular orbital elements. 


\subsection{Relating Relative Orbital Elements to Separation Dis- tances}

The previous sections provided us a means to relate the thruster firings and osculating orbital elements at any point in time. In this section we present a mapping between orbital elements differences and coordinates in the radial, tangential and normal reference frame attached to the leader satellite $\left(x_{r}, x_{t}, x_{n}\right)$. The relative orbital elements are obtained by subtracting the leader element from the follower element, and are indicated by the $\Delta$-symbol. The absolute orbital elements in the equations below refer to the leader satellite. This first order mapping is found in [9], where it was introduced as part of a relative motion model:

$$
\begin{aligned}
& x_{r} \approx-\frac{2}{3} \frac{a}{n} \Delta n-a \Delta e_{x} \cos L-a \Delta e_{y} \sin L \\
& x_{t} \approx a \Delta L+2 a \Delta e_{x} \sin L-2 a \Delta e_{y} \cos L \\
& x_{n} \approx 2 a \Delta i_{x} \sin L-2 a \Delta i_{y} \cos L .
\end{aligned}
$$

This linear mapping between relative orbital elements and the relative Cartesian position in the radial, tangential, normal reference frame is valid for near circular, near equatorial orbits. The mapping is still depending on the leader's state. If required, this dependency can be removed by replacing $a$ by $a_{\text {geo }}, n$ by $n_{\text {geo }}$ and $L$ by $\alpha$ for satellites inside a geostationary slot (i.e. if the mapping is valid between two arbitrary satellites inside the slot, it is also valid between a satellite and its slot center).

As in [10] we write relative eccentricity and inclination vectors in polar notation:

$$
\begin{gathered}
\Delta \mathbf{e}=\left(\begin{array}{c}
\Delta e_{y} \\
\Delta e_{x}
\end{array}\right)=\Delta e\left(\begin{array}{c}
\sin \phi \\
\cos \phi
\end{array}\right) \\
\Delta \mathbf{i}=\left(\begin{array}{c}
\Delta i_{y} \\
\Delta i_{x}
\end{array}\right)=\sin \frac{\Delta i}{2}\left(\begin{array}{c}
\sin \theta \\
\cos \theta
\end{array}\right) \approx \frac{\Delta i}{2}\left(\begin{array}{c}
\sin \theta \\
\cos \theta
\end{array}\right),
\end{gathered}
$$

which allows to write the relative separation in radial, tangential, normal coordinates as:

$$
\begin{aligned}
& x_{r} \approx-\frac{2}{3} \frac{a}{n} \Delta n-a \Delta e \cos (L-\phi) \\
& x_{t} \approx a \Delta L+2 a \Delta e \sin (L-\phi) \\
& x_{n} \approx a \Delta i \sin (L-\theta)
\end{aligned}
$$

These equations directly show the relation between the phasing of relative eccentricity and inclination vectors and corresponding relative motion. The typical coordination strategy for collocated geostationary satellites is the inclination / eccentricity vector separation strategy ([2], [11]). This strategy relies fully on the radial-normal plane to ensure safe separation (based on the observation that estimation and prediction of the tangential position coordinate generally is more erroneous). The key idea behind this strategy is to control the relative eccentricity and inclination vectors such that radial and normal motion are approximately $90^{\circ}$ out-of-phase and thus, normal separation is maximal when radial separation vanishes and vice-versa.
As evident from Eqs. 13, this is the case when relative eccentricity and inclination vectors are parallel. We adopt this strategy and add one further constraint on the relative mean longitude, namely that each follower satellite stays within certain bounds in mean longitude from the leader satellite. This reduces the longitude footprint of the fleet and allows for an increased number of satellites to be collocated in the same slot.

We use a leader/follower architecture for controlling the fleet of collocated satellites. One designated leader satellite is controlled using some desired approach and it is assumed that the controlled leader state trajectory $\mathbf{x}^{L}(t)$ is available to each follower satellite (n.b to obtain a "future" state, the current state is propagated using the latest maneuver plan). The follower satellites are controlled relative to this leader trajectory and are generally assumed to follow this trajectory at a constant offset that is different for each follower.

We performed an error analysis of the linear mapping for the cases analyzed in the remainder of this paper and found that the relative error defined as:

$$
e_{\mathrm{rel}}=\frac{\sqrt{e_{r}^{2}+e_{t}^{2}+e_{n}^{2}}}{\sqrt{x_{r}^{2}+x_{t}^{2}+x_{n}^{2}}},
$$

where $e_{r}, e_{t}, e_{n}$ are the error between the approximate linear and exact nonlinear mapping, was at all times smaller than $1.4 \cdot 10^{-3}$. Note that this statement is valid for the cases analyzed in this paper.

\section{Collocation as a convex optimization problem}

In this section we will extend the approach introduced in [4] to a fleet of collocated satellites with constraints on relative states.

\subsection{Relative state, state error and constraints}

The relative state is simply obtained by subtracting the leader state from the follower state. The leader state $\mathbf{x}^{L}(t)$ is sampled at the discrete time points matching the discretized follower state to obtain $X^{L}$ and the relative state is formed as follows:

$$
\begin{aligned}
\Delta X^{i} & =X^{i}-X^{L} \\
& =\mathbf{F} \mathbf{x}_{0}^{i}+\frac{1}{m^{i}} \mathbf{H} \boldsymbol{\Gamma}^{i} T^{i}+\mathbf{J} D^{i}-X^{L},
\end{aligned}
$$

where $i=1,2,3, \ldots$ refers to the $i^{\text {th }}$ follower satellite. Note that also the term $D$ is different for each satellite in the fleet, as it depends on all dominant perturbations, including solar radiation pressure, which in turn is dependent on the particular satellite's characteristics. Actually, differential perturbations other than solar radiation pressure are very similar for the different satellites, and could be canceled in forming the relative state. We however decided to maintain also these perturbations, as it allows us to evaluate also the absolute state and possibly constrain it. Now 
let $\Delta X_{\text {des }}^{i}$ be the desired relative state of follower satellite $i$, the state error is then defined as:

$$
\begin{aligned}
E^{i} & =\Delta X^{i}-\Delta X_{\mathrm{des}}^{i} \\
& =\mathbf{F} \mathbf{x}_{k}^{i}+\frac{1}{m^{i}} \mathbf{H} \boldsymbol{\Gamma}^{i} T^{i}+\mathbf{J} D^{i}-X^{L}-\Delta X_{\mathrm{des}}^{i},
\end{aligned}
$$

The key task of the collocation control algorithm is to maintain a convex function of this error within certain bounds. In general terms this amounts to:

$$
C_{\mathrm{lb}}^{i} \leq C^{i}\left(E^{i}\right) \leq C_{\mathrm{ub}}^{i}
$$

where $C^{i}\left(E^{i}\right)$ are $M$ state constraint functions, "lb" and "ub" refer to respectively lower and upper bounds.

\subsection{Scaling of the state and control variables}

In the formulation of an optimization problem it is good practice to scale optimization variables and constraints so that they vary in more uniform ranges. Proper scaling enhances robustness and improves converges of the optimization problem [12]. The optimization variables (i.e. thrust) are scaled so that they vary in the range $[0,1]$. This is achieved through applying the following scaling law:

$$
\begin{aligned}
\overline{\mathbf{T}}^{i} & =\mathcal{T}^{i} \mathbf{T}^{i} \\
& =\left(\begin{array}{llll}
1 / T_{1, \text { max }}^{i} & & & \\
& 1 / T_{2, \max }^{i} & 1 / T_{3, \max }^{i} & \\
& & & 1 / T_{4, \text { max }}^{i}
\end{array}\right) \mathbf{T}^{i}
\end{aligned}
$$

with $T_{\max }$ denotes the (maximum) thrust that a particular thruster can deliver. The complete (concatenated) vector of thrusts in the range $k=1, . ., N$ is defined as:

$$
\begin{aligned}
\bar{T}^{i} & =\mathcal{T}^{i} T^{i} \\
& =\left(\begin{array}{lll}
\mathcal{T}^{i} & & \\
& \mathcal{T}^{i} & \\
& & \ddots
\end{array}\right) T^{i}
\end{aligned}
$$

A similar approach is applied to the constraints. In this work we only consider constraints that have symmetric bounds (i.e. $C_{\mathrm{lb}}^{i}=-C_{\mathrm{ub}}^{i}$ ) or constraints that have a lower bound equal to zero. If $c_{j}^{i}$ denotes the $\mathrm{j}^{\text {th }}$ element of $C^{i}$, we scale that constraint by $1 / c_{j, \mathrm{ub}}^{i}$, so that the scaled constraint $\bar{c}_{j}^{i}$ varies in the range $[0,1]$ or $[-1,1]$, for respectively $c_{j, \mathrm{lb}}^{i}=0$ and $c_{j, \mathrm{lb}}^{i}=-c_{j, \mathrm{ub}}^{i}$. Similar to the thrust vector scaling, we define a diagonal scaling matrix for the constraints:

$$
\begin{aligned}
\bar{C}^{i} & =\mathcal{C}^{i} C^{i} \\
& =\left(\begin{array}{lll}
1 / c_{j, \mathrm{ub}}^{i} & & \\
& 1 / c_{j, \mathrm{ub}}^{i} & \\
& & \ddots
\end{array}\right) C^{i} .
\end{aligned}
$$

These scaled variables are used in the formulation of the optimization problem in the next section.

\subsection{Optimization Problem Formulation}

An optimization based method is used to determine the station-keeping maneuvers of the follower satellites. This section details the formulation of the optimization problem. To solve the collocation control problem for follower satellite $i$ we specify the following optimization problem:

$\operatorname{minimize} \quad \alpha\left\|\bar{T}^{i}\right\|_{1}+(1-\alpha) \sum_{j=1}^{M} \max \left(0, s_{j}-1\right)$

subject to

$$
\begin{aligned}
\left|\bar{C}^{i}\right| & \leq S \\
\bar{T}^{i} & \geq \mathbf{0} \\
\bar{T}^{i} & \leq \mathbf{1}
\end{aligned}
$$

The optimization variables in this problem are 1 . the scaled thrust vector $\bar{T}^{i}$, and 2 . a slack variable vector $S$, which has length of $M$, equal to the number of constraints $\bar{C}^{i}$.

\subsubsection{Cost function}

The cost function contains two terms, the first is the $\ell 1-$ norm of the thrust vector. This term represents the minimization of propellant consumption. The second term is a deadzone-linear penalty function [13], which can be interpreted as follows; whenever the slack variable is smaller (in absolute terms) than the tolerable bound on the constraint function $c_{j, \mathrm{ub}}^{i}$, it is free of charge. Whenever the bound gets violated, it is penalized through the cost function. This implementation gives the optimizer an option to violate desired control windows on state variables at a cost. The advantage of this implementation is that the optimization problem does not become infeasible when the constraints cannot be met. A relative weighting between the two terms in the cost function is added through $\alpha$ (with $\alpha \in[0,1])$.

\subsubsection{Constraints}

Three types of state constraints were used in this work. The first two constraint types are bounds on the $\ell 2$-norm of the eccentricity and inclination vector error $\left(\Delta e_{x}, \Delta e_{y}\right)^{T}$ and $\left(\Delta i_{x}, \Delta i_{y}\right)^{T}$. To arrive at a specific example, let $E_{j, k}^{i}$ denote the $j^{\text {th }}$ relative state error at discrete time $k$. A constraint on the $\ell 2$-norm of the eccentricity vector error at time $k$, including scaling and slack variable is as follows:

$$
\frac{1}{c_{j, \mathrm{ub}}^{i}}\left\|\begin{array}{c}
E_{2, k}^{i} \\
E_{3, k}^{i}
\end{array}\right\|_{2} \leq s_{j}
$$

and on the inclination vector error:

$$
\frac{1}{c_{j, \mathrm{ub}}^{i}}\left\|\begin{array}{c}
E_{4, k}^{i} \\
E_{5, k}^{i}
\end{array}\right\|_{2} \leq s_{j}
$$


The third type of constraint is a box constraint on the longitude error $\Delta L$, implemented as:

$$
\frac{1}{c_{j, \mathrm{ub}}^{i}}\left|E_{6, k}^{i}\right| \leq s_{j}
$$

The constraint on the control variables $\bar{T}$ are bounds on the maximum thrust force that the satellite can deliver. After applying the scaling, each element in $\bar{T}$ is bounded between 0 and 1 .

\section{Simulation and Analysis}

In this section we present the simulation environment and the two analysis cases used to evaluate the presented method for controlling collocated satellites.

\subsection{Simulation environment}

Simulations were carried out using Matlab. We used a validated propagator including Earth gravity up to $8^{\text {th }}$ order and degree, Moon gravity, Sun gravity and solar radiation pressure (SRP). The RK4 integration method was used, with a timestep of 100 seconds. The formulation of the optimization problem was done using CVX [14], and solved using MOSEK [15]. The timestep in the optimization problem was 1000 s. Maneuver plans were implemented based on simple on/off thruster with a single operational point (i.e. $T=0$ or $T=T_{\max }$ ) as in [4]. Several forms of uncertainty were included; Gaussian orbit determination errors were implemented based on the covariance matrix in Table 1, actuator uncertainty was included by implementing a 5\% thrust force error (Gaussian, $3 \sigma$ ) and a $1.5^{\circ}$ attitude error (Gaussian, $3 \sigma$ ). In addition, SRP uncertainty was included as a $15 \%$ uniform random error on the acceleration due to SRP.

\subsection{Analysis cases}

We present two concrete example cases of a fleet of satellites controlled in geostationary slots of respectively $\pm 0.1^{\circ}$ and $\pm 0.05^{\circ}$, located at $19.2^{\circ} \mathrm{E}$. In the first case, the leader satellite is controlled using a sun-pointing perigee strategy, while in the second case the mean eccentricity is controlled to a fixed point. The leader's inclination control strategy is such that only secular variations of the inclination vector are compensated, while the periodic oscillations are uncontrolled. The longitude control strategy targets the center of the slot at the end of the maneuver cycle in both cases. The follower satellites are then controlled relative to the leader satellite using the method outlined in the previous section.

\subsubsection{Case A: a fleet of four satellites}

With this simulation case we aim to show that the method is robust under realistic (even conservative) assumptions on uncertainty, for long duration maneuver cycles, for an inhomogeneous fleet. We aim to show that the

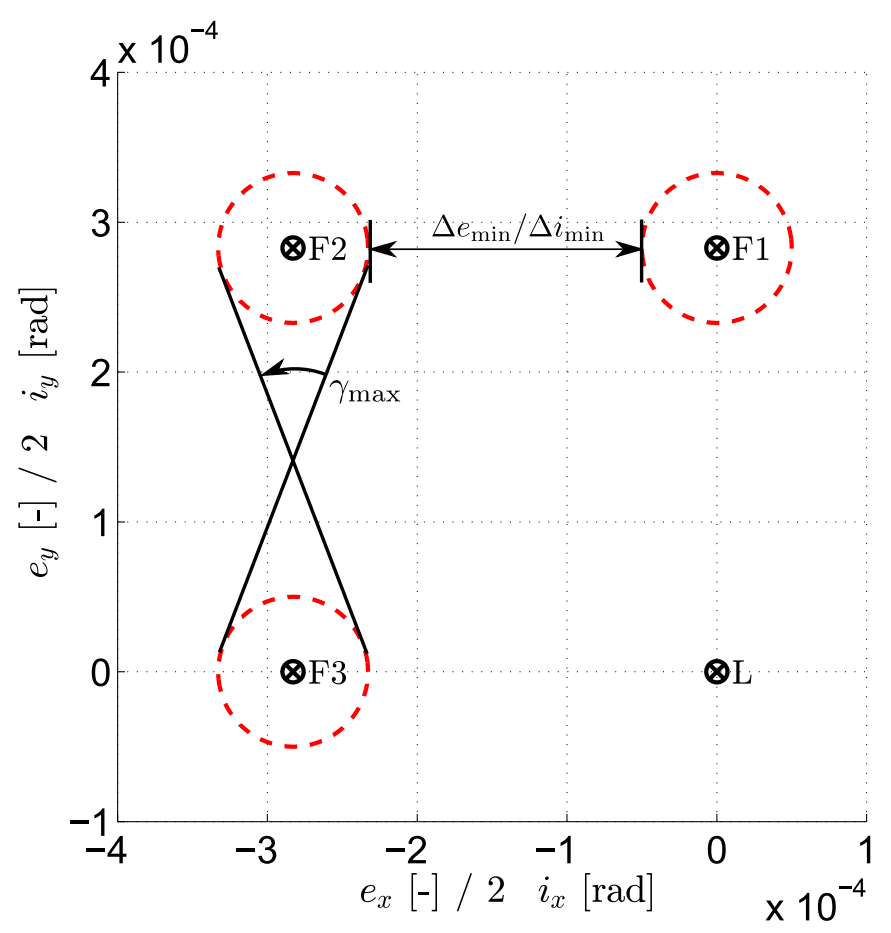

Figure 2: Minimum relative eccentricity and inclination and maximum phasing angle between relative inclination and eccentricity vectors

method has no significant penalties in performance, while maintaining safe separation distances. Performance is measured in terms of propellant consumption and thruster firings.

The fleet consists of four satellites. The follower satellites have characteristics different from the leader satellite in terms of area exposed to the Sun, maximum thrust force and minimum impulse bit. Between the three follower satellites the characteristics are identical, with the exception of their thruster configuration. The satellite characteristics are summarized in Table 2. The maneuver plans for the follower satellites are determined with a prediction horizon of 7 days, in line with usual operational practice, which are then executed open-loop. The leader follows a Sun-pointing perigee strategy, following a circle in the eccentricity plane with a radius of $2 \cdot 10^{-4}$, the eccentricity circle's center and other nominal leader parameters are given in Table 3 .

Table 4 shows the bounds that were implemented in the optimization problem. Figure 2 graphically shows the eccentricity and inclination vector configuration. The nominal relative states of the follower satellites were chosen consistent with the eccentricity inclination vector separation strategy. The dashed line shows the bounds in the optimization problem. The bounds on relative eccentricity and inclination vectors are also bounds on the minimum relative eccentricity and inclination, and also a bound on the maximum phasing angle between relative eccentricity and inclination vectors, as seen from the figure.

Using Eqs. 13 we can obtain a bound on the minimum 
Table 1: Covariance matrix of orbit determination error, Radial (R), Tangential (T) and Normal (N) position and velocity (V)

\begin{tabular}{lrrrrrr}
\hline & $\mathrm{R}$ & \multicolumn{1}{c}{$\mathrm{T}$} & $\mathrm{N}$ & $\mathrm{VR}$ & $\mathrm{VT}$ & \multicolumn{1}{c}{$\mathrm{VN}$} \\
\hline $\mathrm{R}$ & $1.23 \mathrm{E}+01$ & $4.90 \mathrm{E}+01$ & $1.12 \mathrm{E}+01$ & $2.95 \mathrm{E}-04$ & $-1.70 \mathrm{E}-03$ & $-1.94 \mathrm{E}-03$ \\
$\mathrm{~T}$ & $4.90 \mathrm{E}+01$ & $1.47 \mathrm{E}+05$ & $-1.56 \mathrm{E}+02$ & $2.09 \mathrm{E}-03$ & $-7.86 \mathrm{E}-03$ & $2.74 \mathrm{E}-02$ \\
$\mathrm{~N}$ & $1.12 \mathrm{E}+01$ & $-1.56 \mathrm{E}+02$ & $1.32 \mathrm{E}+02$ & $2.31 \mathrm{E}-04$ & $-1.50 \mathrm{E}-03$ & $-2.30 \mathrm{E}-02$ \\
$\mathrm{VR}$ & $2.95 \mathrm{E}-04$ & $2.09 \mathrm{E}-03$ & $2.31 \mathrm{E}-04$ & $9.20 \mathrm{E}-09$ & $-4.33 \mathrm{E}-08$ & $-4.02 \mathrm{E}-08$ \\
$\mathrm{VT}$ & $-1.70 \mathrm{E}-03$ & $-7.86 \mathrm{E}-03$ & $-1.50 \mathrm{E}-03$ & $-4.33 \mathrm{E}-08$ & $2.37 \mathrm{E}-07$ & $2.61 \mathrm{E}-07$ \\
$\mathrm{VN}$ & $-1.94 \mathrm{E}-03$ & $2.74 \mathrm{E}-02$ & $-2.30 \mathrm{E}-02$ & $-4.02 \mathrm{E}-08$ & $2.61 \mathrm{E}-07$ & $4.00 \mathrm{E}-06$ \\
\hline
\end{tabular}

separation in the radial-normal plane. Assuming that the semi-major axis difference is small, we have:

$$
d^{2}=a^{2} \Delta e^{2} \cos ^{2}(L-\phi)+a^{2} \Delta i^{2} \sin ^{2}(L-\theta)
$$

If we define $\lambda=L-\phi$ and $\gamma=\phi-\theta$ as the phasing angle between relative eccentricity and inclination vectors, we obtain:

$$
d^{2}=a^{2} \Delta e^{2} \cos ^{2}(\lambda)+a^{2} \Delta i^{2} \sin ^{2}(\lambda-\gamma)
$$

with

$$
\gamma=\cos ^{-1}\left(\frac{(\Delta \mathbf{e})^{T}(\Delta \mathbf{i})}{\Delta e \cdot \Delta i}\right)
$$

and $\lambda \in[0,2 \pi]$. The largest possible phasing angle for relative eccentricity and inclination vector inside boundary $\gamma_{\max }$ is $41.4^{\circ}$ (see Figure 2). The minimum relative eccentricity and inclination vector can also be determined from Figure 2 and are both equal $1.83 \cdot 10^{-4}$ (rad). Using these values allows us to calculate a quick but conservative worst-case minimum separation distance. However, since the minimum relative eccentricity and inclination vectors and phasing angle are not independent, $\Delta e_{\min }$ and $\Delta i_{\min }$ and $\gamma_{\max }$ cannot occur simultaneously. Using their dependency, together with Eqs. 13 allows to minimize the smallest separation distance over all possible combinations of $\Delta \mathbf{e}$ and $\Delta \mathbf{i}$ satisfying the constraints. This is in itself an optimization problem and can be solved to yield the absolute worst-case minimum separation distance (in the radial-normal plane):

$$
d_{\min } \geq 5.97 \mathrm{~km}
$$

which occurs at $\gamma=36.84^{\circ}$, with $\Delta e=\Delta i=2.24 \cdot 10^{-4}$. Thus maintaining the relative eccentricity and inclination vectors within the specified convex bounds will guarantee a minimum separation distance which is by no means a convex constraint in Cartesian space.

Note that due to the slack variable and the deadzone linear term in the cost function the constraints are not hard bounds, instead, they could be violated. We found that especially in the presence of errors (orbit determination, actuators, modeling) performance increases when we implemented a tighter bound on the final state. We also found that it is good practice to make the satellite with the smallest acceleration the formation leader. Key reason is that a formation leader with a significantly more capable propulsion system could produce reference trajectories which cannot be realized by satellite with a less capable

\begin{tabular}{|c|c|c|}
\hline & Case A & Case B \\
\hline$e_{x}[-]$ & $-1.41 \mathrm{E}-04$ & $-0.60 \mathrm{E}-04$ \\
\hline$e_{y}[-]$ & $1.41 \mathrm{E}-04$ & $0.60 \mathrm{E}-04$ \\
\hline$i_{x}[\mathrm{rad}]$ & $-0.71 \mathrm{E}-04$ & $-0.30 \mathrm{E}-04$ \\
\hline$i_{y}[\mathrm{rad}]$ & $0.71 \mathrm{E}-04$ & $0.30 \mathrm{E}-04$ \\
\hline$\Delta L[\mathrm{rad}]$ & 0 & 0 \\
\hline
\end{tabular}
propulsion system.

Table 2: Parameters of four satellites

\begin{tabular}{rcccc} 
Table 2: Parameters of four satellites \\
\hline Parameter & L & 1 & 2 & 3 \\
\hline Mass [kg] & 3000 & 3000 & 3000 & 3000 \\
Surface Area [m $\left.{ }^{2}\right]$ & 90 & 120 & 120 & 120 \\
Reflection coefficient [-] & 1.2 & 1.2 & 1.2 & 1.2 \\
Thrust force [mN] & 75 & 125 & 125 & 125 \\
Min. Impulse [Ns] & 7.5 & 12.5 & 12.5 & 12.5 \\
Thruster configuration & REF & REF & A & B \\
\hline
\end{tabular}

Table 4: Bounds on different state constraints

\begin{tabular}{rllll}
\multicolumn{6}{c}{ Table 4: Bounds on different state constraints } \\
\hline \multicolumn{4}{c}{ Case A } & \multicolumn{3}{c}{ Case B } \\
$c_{j, \mathrm{ub}}^{i}$ & at $k<N$ & at $k=N$ & at $k<N$ & at $k=N$ \\
\hline$\Delta e[-]$ & $5.00 \mathrm{E}-05$ & $2.50 \mathrm{E}-05$ & $2.50 \mathrm{E}-05$ & $1.25 \mathrm{E}-05$ \\
$\Delta i[\mathrm{rad}]$ & $5.00 \mathrm{E}-05$ & $2.50 \mathrm{E}-05$ & $2.50 \mathrm{E}-05$ & $1.25 \mathrm{E}-05$ \\
$\Delta L[\mathrm{rad}]$ & $1.00 \mathrm{E}-04$ & $5.00 \mathrm{E}-05$ & $1.00 \mathrm{E}-04$ & $5.00 \mathrm{E}-05$ \\
\hline
\end{tabular}

\subsubsection{Case B: a fleet of 16 satellites}

If we desire to control the relative orbital elements within tighter bounds, under the assumed errors in orbit determination, actuation and modeling, we can only do so by increasing the frequency of re-planning. To avoid unnecessary burden on the operator, the method can and should be implemented in a fully automated manner. A necessary element to achieve this is an automated orbit determination capability, which can e.g. be enabled by 
an on-board GNSS receiver, see e.g [16], containing acceptance testing performance results for a GPS receiver in GEO. Their results are significantly better than the orbit determination uncertainty assumed in this work. Assuming such capability, we investigated the collocation of a fleet of 16 satellites in a $\pm 0.5^{\circ}$ slot, using the proposed method. The leader's nominal mean orbital elements are given in Table 3 . The nominal inclination and eccentricity vector configuration can be easily derived from Figures 6 and 7. In this case we assumed identical satellites, with thruster configuration B. The satellite characteristics are identical to follower 3 in case A (see Table 2). Making a similar analysis as in case A, we can guarantee minimum separation distances in the radial-normal plane larger than $2.08 \mathrm{~km}$. We further aim to maintain performance in terms of propellant consumption and number of maneuvers, despite of the much shorter maneuver cycle. Note that this case significantly improves the state-of-art as currently up to 8 satellites have been collocated in a slot that is twice the size in longitude $\left( \pm 0.1^{\circ}\right)$.

\section{Results and Discussion}

\subsection{Case $A$}

Figures 3 to 5 show the results of case A for respectively relative eccentricity control, relative inclination control and relative mean longitude control. The striped lines indicate the soft constraints as formulated in the optimization problem. Table 5 contains results in terms of propellant consumption (measured in $\Delta V$ ) and number of thruster firings.

A geostationary satellite with a low thrust propulsion system usually requires roughly two North-South and two East-West maneuvers per day, which, depending on the thruster configuration can be realized with 4 to 8 burns. Hence, the expected number of burns lies somewhere between 1460 and 2920 per year and anything in this range is deemed acceptable. In terms of $\Delta V$ we expect roughly 50 $\mathrm{m} / \mathrm{s}$ per year for the classical thruster configuration and $71 \mathrm{~m} / \mathrm{s}$ for the other configurations (approximately $95 \%$ of thrust is required in North-South direction, and configuration $\mathrm{A}$ and $\mathrm{B}$ thus have a cosine loss equal to $\sqrt{2}$ in that direction).

The results in terms of $\Delta V$ and number of firings are very much in line with expectations. We note that by explicitly taking the thruster configuration into account in the optimization problem, we saved $2-4 \mathrm{~m} / \mathrm{s} \Delta V$ for configuration $\mathrm{A}$ and $\mathrm{B}$. Note that in case of configuration $\mathrm{A}$, we could optimize for North-South and East-West burns and realize each burn by simultaneous firing of two thrusters. The resulting $\Delta V$ could be approximately equal to that of Follower 1 , multiplied by $\sqrt{2}$. We could not deal with configuration B at all without explicitly accounting for the thruster configuration as part of the optimization problem.

We observe that follower 1 required a bit more propellant than the leader satellite, despite of having the same thruster configuration. Reason is the higher solar radiation pressure on follower 1. Furthermore, every follower required less thruster pulses as the formation leader. Reason for this is that the follower satellites are controlled within tolerance windows, while the leader satellite was commanded to exact coordinates at the end of each maneuver cycle.

A very interesting observation is the importance of the thruster configuration on the control accuracy. We observe that the eccentricity and longitude control are significantly degraded for thruster configuration A. The key reason for this behavior is that both thrust force errors and attitude errors for every firing can have significant components in East/West direction. Since both the eccentricity and longitude are very sensitive to East/West thrust errors, we observe much larger errors in these elements. In configuration $\mathrm{B}$ this problem is much smaller, since the East-West component of thrust is very small, and hence, errors in the thrust force have almost no component in East-West direction.

We also analyzed the minimum separation distance in the radial-normal plane, for every satellite pair (6 combinations). We found that the minimum separation distance was equal to $7.30 \mathrm{~km}$ over the full one year simulation. Thus even though we had some significant violations of the constraint windows, especially thruster configuration $\mathrm{A}$, the achieved minimum separation distance was well within acceptable range.

The constraints, especially for the eccentricity vector, were violated significantly for thruster configuration A (although only for a very brief period of time). Despite of that, a sufficient separation distance was achieved and the results in terms of propellant consumption and thruster firings were also very much within acceptable range. We note however, that to achieve better accuracy, we can simply reduce the maneuver cycle duration. This is demonstrated in case B.

\begin{tabular}{|c|c|c|c|c|}
\hline & $\mathrm{L}$ & 1 & 2 & 3 \\
\hline$\Delta V[\mathrm{~m} / \mathrm{s}]$ & 50.8 & 52.2 & 69.9 & 69.2 \\
\hline Pulses & 2184 & 1494 & 1879 & 1844 \\
\hline
\end{tabular}

\subsection{Case $B$}

Figures 6 and 7 show the results of case B. The results show only minor violations of the control windows (which were much smaller than the control windows in case A). We further analyzed whether no satellite left the geostationary slot. We found that all satellites remained within the longitude window $\left[-0.046^{\circ}, 0.049^{\circ}\right]$. The minimum distance in the radial-normal plane among all 120 pairs was equal to $3.39 \mathrm{~km}$. We then looked into the difference between the trajectory as calculated by the optimizer (including effects of modeling errors, orbit determination errors and 


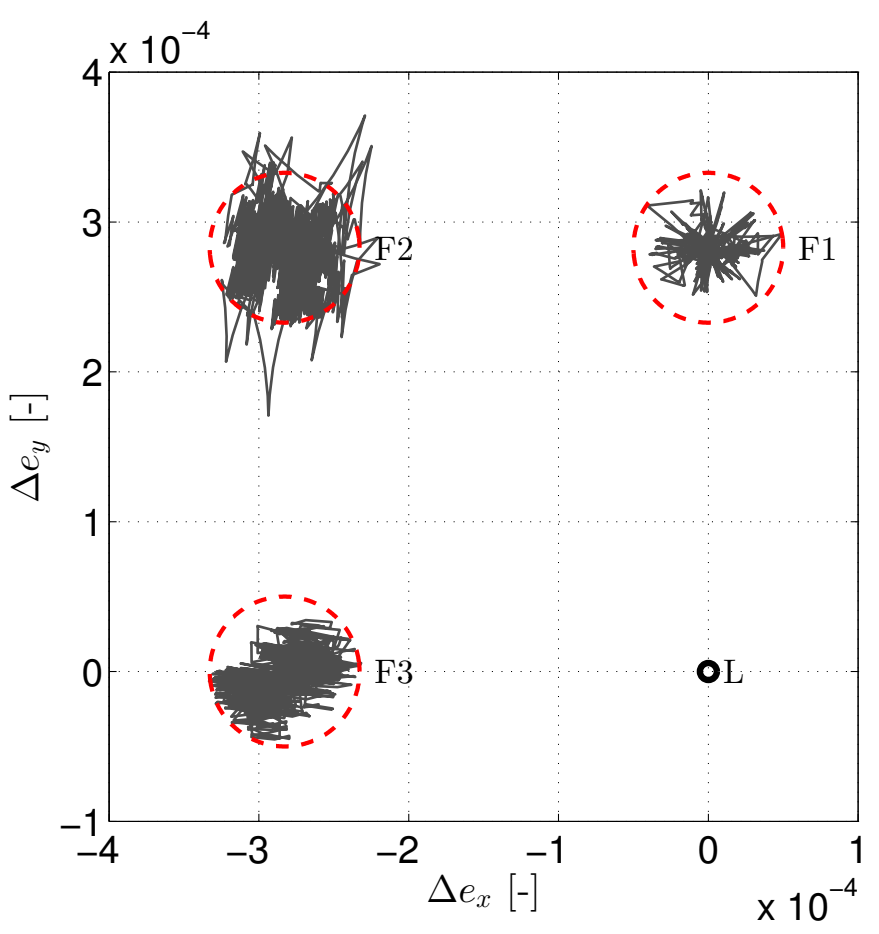

Figure 3: Eccentricity vector, horizon 7 days, including errors

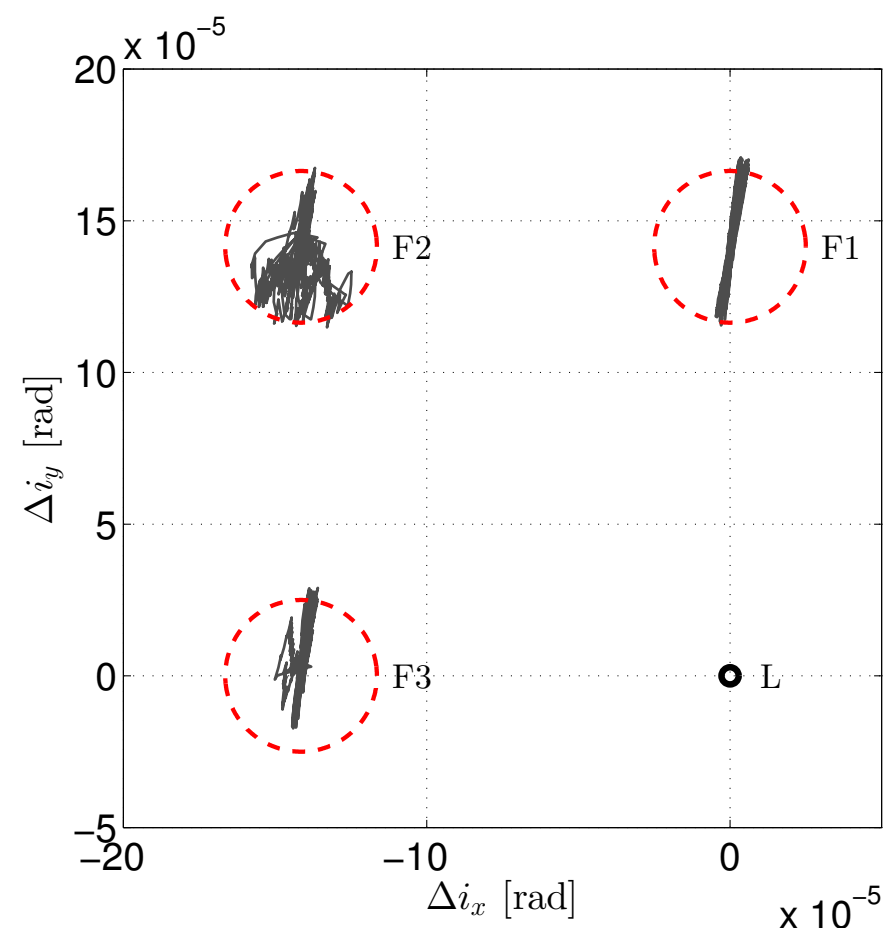

Figure 4: Inclination vector, horizon 7 days, including errors

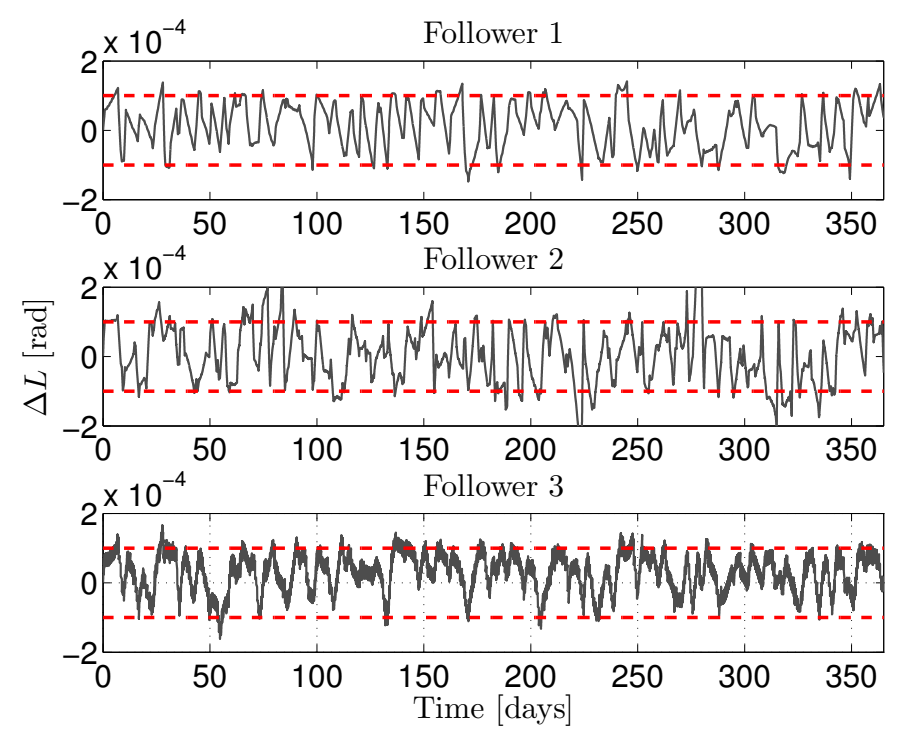

Figure 5: $\Delta L$, horizon 7 days, including errors

actuator errors) and the actual trajectory actually flown and found the following maximum errors over the complete one year simulation, over all satellites:

- Radial error: $175.5 \mathrm{~m}$

- Tangential error: $1835 \mathrm{~m}$

- Normal error: $88.7 \mathrm{~m}$

This further confirms that it is a good choice to ensure safe separation using only the radial-normal plane. The errors between predicted and actual trajectories are very acceptable and the combined radial, normal error is far smaller than the achieved minimum separation distances, allowing us to conclude that the strategy is safe, even with 16 satellites in a small geostationary slot.

The average $\Delta V$ required for controlling the fleet was equal to $69.2 \mathrm{~m} / \mathrm{s}$ and the average number of thruster pulses was equal to 2507 pulses. Since no sun-pointing perigee was followed in this scenario, an increase in $\Delta V$ might have been expected. The results show however no increase in $\Delta V$. This can be explained because the satellites require much more $\Delta V$ in $\mathrm{N} / \mathrm{S}$ direction than $\mathrm{E} / \mathrm{W}$ direction and since the thrusters are inclined with respect to the N/S plane, every thruster firing basically gives a "free" E/W thrust. By accounting for the thruster configuration in the optimization problem we were able to use this to our benefit. Thus for satellites with typical electric propulsion thruster configurations, a sun-pointing perigee strategy is not required to save propellant. This leaves more space in the eccentricity vector plane, and allows to control more satellites within a single slot.

\section{Conclusion}

We have shown that relative orbital elements provide a direct measure for relative distances of geostationary 


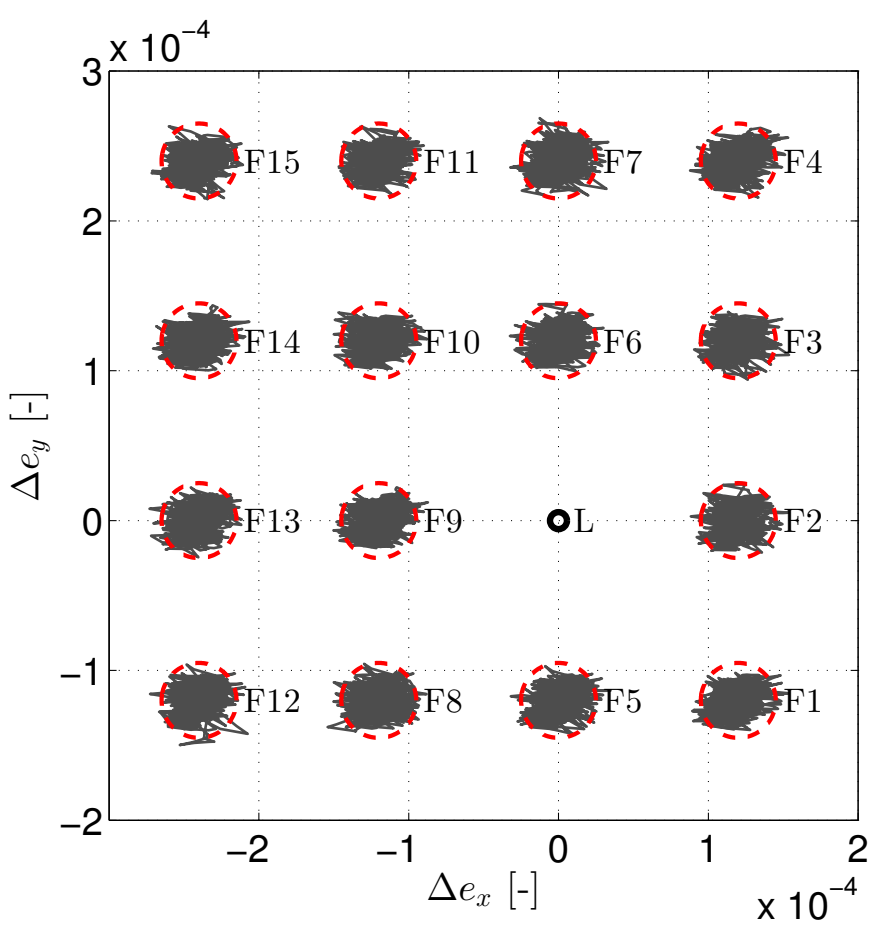

Figure 6: Relative eccentricity vector, horizon 1 day, including errors

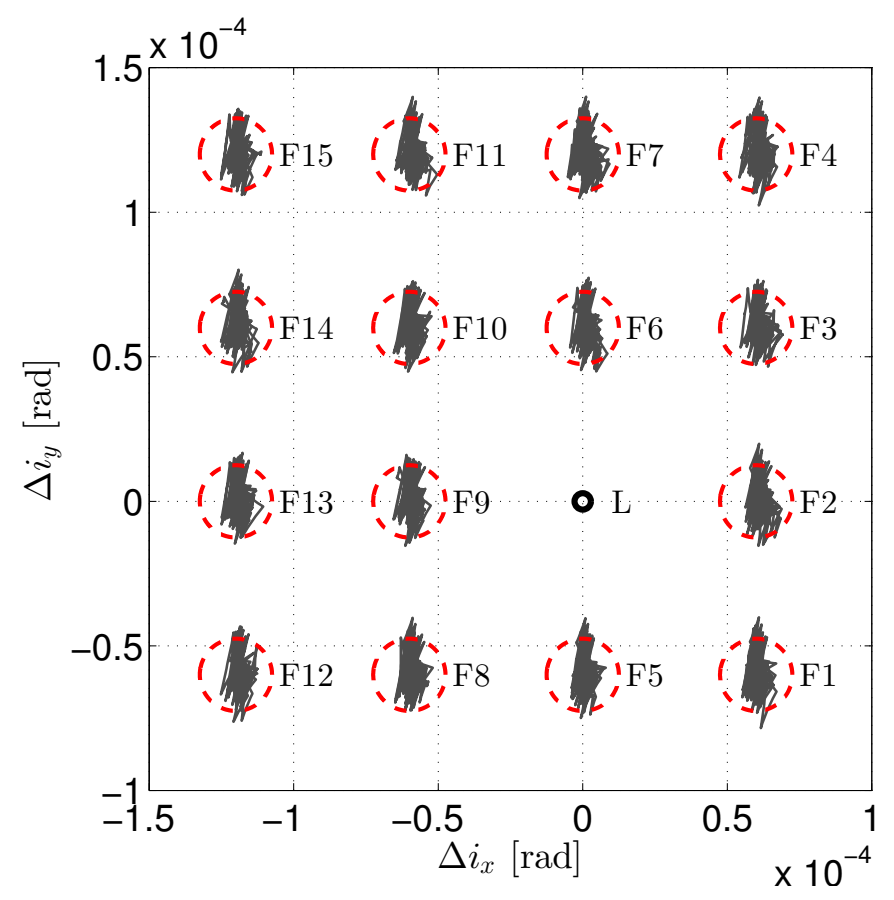

Figure 7: Relative inclination vector, horizon 1 day, including errors satellites. By controlling relative orbital elements within certain tolerance windows we can guarantee safe separation distances between satellites. These relative orbital elements are thus well-suited for controlling a fleet of satellites.

We formulated the (relative) station-keeping problem of a fleet of collocated satellites as a convex optimization problem, using a leader / follower control hierarchy. Using the method developed in this paper we were able to control directly the satellites' relative osculating orbital elements.

The method was demonstrated to work for an inhomogeneous fleet, under realistic (even conservative) errors in orbit determination, actuation and modeling for maneuver cycles of one week. We have shown that significant advantages are obtained for shorter maneuver cycles and demonstrated that we can control 16 satellites in a $\pm 0.05^{\circ}$ longitude slot, while maintaining safe separation distances and with good performance in terms of thruster firings and propellant consumption. We emphasized that the presented method is most beneficial with an automated implementation and an automated, ideally on-board, orbit determination capability is key in realizing collocation of a large fleet efficiently.

We further demonstrated that by including the thruster configuration explicitly in the optimization problem, a small propellant saving is achieved. Furthermore, we showed that for satellites with typical electric propulsion thruster configurations the classic sun-pointing perigee strategy brings no significant advantages in terms of propellant savings and only limits the eccentricity vector configuration space. Lastly, the thruster configuration has an impact on the achieved control accuracy and an exemplary configuration with good performance was introduced.

\section{References}

[1] P. Wauthier, P. Francken, H. Laroche, Co-location of six astra satellites: Assessment after one year of operations, in: Proceedings of the $12^{\text {th }}$ International Symposium on Space Flight Dynamics, European Space Agency, 1997, p. 13.

[2] M. Eckstein, C. Rajasingh, P. Blumer, Colocation strategy and collision avoidance for the geostationary satellites at 19 degrees west, in: International Symposium on Space Flight Dynamics, 1989.

[3] P. Wauthier, P. Francken, The ASTRA co-location strategy for three to six satellites, Revista Brasileira de Ciencias Mecanicas (ISSN 0100-7386), vol. 16, p. 163-171 16 (1994) 163-171.

[4] F. de Bruijn, D. Choukroun, E. Gill, S. Theil, Geostationary satellite station-keeping using convex optimization, Submitted to JGCD.

[5] M. Eckstein, Geostationary orbit control considering deterministic cross coupling effects, in: Proceedings of the $41^{\text {st }}$ International Astronautical Federation (IAF) Congress, IAF, 1990.

[6] O. Montenbruck, E. Gill, Satellite Orbits, Springer Berlin Heidelberg, 2000. doi:10.1007/978-3-642-58351-3. URL http://dx.doi.org/10.1007/978-3-642-58351-3

[7] H. Schaub, J. Junkins, Analytical Mechanics Of Space Systems, American Institute of Aeronautics and Astronautics, 2003. doi:10.2514/4.861550. URL http://dx.doi.org/10.2514/4.861550

[8] C. Chasset, P. Bodin, N. Neumann, R. Larsson, A. Edfors, Evolution of the AOCS design for EDRS and the satellites of the 
Small GEO family, in: Proceedings of the $9^{\text {th }}$ International ESA Conference on Guidance, Navigation and Control, ESA, 2014.

URL http://www.esa-gnc.eu/

[9] P. BLUMER, A future concept of coordinated orbit control of colocated geostationary satellites, in: Astrodynamics Conference, American Institute of Aeronautics and Astronautics (AIAA), 1992. doi:10.2514/6.1992-4654.

URL http://dx.doi.org/10.2514/6.1992-4654

[10] D'Amico, S, Autonomous Formation Flying in Low Earth Orbit, Ph.D. thesis, Delft University of Technology (2010).

[11] E. M. Soop, Handbook of Geostationary Orbits, Microcosm and Kluwer, 1994. doi:10.1007/978-94-015-8352-7.

URL http://dx.doi.org/10.1007/978-94-015-8352-7

[12] J. Betts, Very low-thrust trajectory optimization using a direct $\{\mathrm{sqp}\}$ method, Journal of Computational and Applied Mathematics 120 (1-2) (2000) 27-40. doi:10.1016/s03770427(00)00301-0.

URL http://dx.doi.org/10.1016/s0377-0427(00)00301-0

[13] S. Boyd, L. Vandenberghe, Convex Optimization, Cambridge University Press, 2004. doi:10.1017/cbo9780511804441.

URL http://dx.doi.org/10.1017/cbo9780511804441

[14] M. Grant, S. Boyd, CVX: Matlab software for disciplined convex programming, version 2.0 beta, http://cvxr.com/cvx (sep 2013).

[15] E. Andersen, B. Jensen, J. Jensen, R. Sandvik, U. Worsøe, Mosek version 6, Tech. rep., Technical Report TR-2009-3, MOSEK (2009).

[16] J. Chapel, D. Stancliffe, T. Bevacqua, S. Winkler, B. Clapp, T. Rood, D. Gaylor, D. Freesland, A. Krimchansky, Guidance, navigation, and control performance for the GOES-r spacecraft, CEAS Space Jdoi:10.1007/s12567-015-0077-1.

URL http://dx.doi.org/10.1007/s12567-015-0077-1 